\title{
Performance Analysis of Various Architectural Approaches in Cloud Computing Environment for Energy and Bandwidth Minimization
}

\author{
N.R. Ram Mohan \\ Research Scholar, Computer and Information Tech., \\ Manonmaniam Sundaranar University, \\ Tirunelveli, Tamilnadu, India
}

\author{
E. Baburaj \\ Professor, Dept of Computer Science and \\ Engineering, \\ Sun College of Engineering and Technology, \\ Nagercoil, Tamilnadu, India
}

\begin{abstract}
Cloud Computing is one of the mainly admired subject in the computational world. It is support to processing the data that was emerged by profitable infrastructure. Cloud computing consist of large number of servers comprising of both virtual and physical servers in order to provide the resources in an optimal manner. The evolution of Cloud computing provides customers the illusion of infinite computing resources which are available from anywhere, anytime, on demand. It offers a user the service (called "Infrastructure as a Service" - IaaS) of renting computing resources over the Internet. Some of the important issues related to cloud computing are the cloud rely on large scale infrastructures, consumes maximum bandwidth and high energy consumption to obtain the process. The user can select from different types of computing resources based on the requirements. In this work, we have evaluated several existing cloud computing techniques related on energy and bandwidth consumption. Various Energy conservation strategies and resource allocation strategies and their challenges are discussed for which the results obtained can be benefitted by both researchers and cloud users. The work also evaluates the impact created by the resource while performing scheduling during various aspects, including number of users involved in cloud, types of resources used and the total number of data centers involved while performing the analysis. The result of survey not only measures the similarities and differences of the different architectural approaches presented for cloud users but also to identify areas requiring further research.
\end{abstract}

\section{Index Terms}

Cloud Computing, Energy consumption, Bandwidth Consumption

\section{INTRODUCTION}

TODAY, the most popular applications are Internet services with millions of users. Websites like Google, Yahoo and Facebook receive millions of clicks daily. This results in terabytes of unusable data which can be further improvised for performing online advertising strategies and user satisfaction. Real time capturing, storage, and analysis of this data are common needs of all high-end online applications. To address these problems, a number of cloud computing technologies have emerged in last few years. Cloud computing is a type of computation which provides with the scalability issues and resources provided in virtualized manner as a service over the Internet. The cloud refers to the datacenter hardware and software that supports a clients needs, often in the form of data stores and remotely hosted applications. These infrastructures enable companies to cut costs by eliminating the need for physical hardware, allowing companies to outsource data and computations on demand. The cloud developers with novel concepts for providing services in Internet do not require higher capital investment related to the hardware devices in order to design and implement their services. This is one of the major shifts towards IT paradigm. The processing of large scale, computer related to commodity datacenters was the key principle behind cloud computing, due to the fact that these datacenters take advantage of economies of scale, resulting in the minimization of electricity cost involved in cloud, the bandwidth requirements, operational performance and hardware requirements.

The cloud computing advantages are, lower costs, reprovisioning of resources and remote accessibility. Cloud computing lowers cost by avoiding the capital expenditure by the company in renting the physical infrastructure from a third party provider. Due to the flexible nature of cloud computing, we can quickly access more resources from cloud providers when we need to expand our business. The remote accessibility enables us to access the cloud services from anywhere at any time. In order to solve the problem s discussed above, the services offered in terms of resources should be allocated optimally to the applications running in the cloud. The following section discusses the significance of resource allocation.

Resource Allocation Strategy (RAS) is the process of integrating the cloud provider activities for utilizing and allocating scarce resources. Within that the limitation of cloud environment, that was to meet the needs of the cloud application. It requires the type and amount of resources needed by each application in order to complete a user job. The order and time of allocation of resources are also an input for an optimal RAS. Resource providers' allocation of resources may lead to an underprovisioning of resources. To overcome the above mentioned problems, inputs needed from both cloud providers and users for a RAS [18].Some of the inputs required from the other aspects include, status about the resources being maintained, the resource availability in order to manage and allocate resources to host applications by RAS. The output generated using RAS should satisfy the following parametric considerations such as throughput, latency and response time. The complexity of finding an optimum resource allocation is exponential in huge systems like big clusters, data centers or Grids. The proposed technique are resource demand and supply can be dynamic and uncertain, various strategies for resource allocation. This work puts forth various resource allocation strategies deployed in cloud environments.

The rest of the work is organized as follows: In section 2, literature review is presented from different angles from the point of numerous researchers performed related to resource allocation 
in cloud environment. Section 3 discusses about the different resource allocation strategies and their effects in cloud environments in an elaborate manner. Section 4 discusses in detail about the performance evaluation of different allocation strategies. Experimental results are discussed in section 5. Section 6 details about the research direction. Finally the conclusion of the work is elaborated in section 7 .

\section{LITERATURE REVIEW}

Resource allocation is cloud is one of the major problems to be solved in cloud computing. Several researchers have conducted different methods for it. In [17], face the challenge in automated calibration of resource allocation for parallel processing of analytic tasks. The proposed framework does not assume availability of data statistics and application semantics. In [3], different policies for resource allocation in cloud were provided on the basis of service-level-agreement, decision-making in centralized manner and multiple criteria decision making. In [5], the author provides the solution for integrity of storage of data in cloud environment. In [15], Cooperative Provable Data Possession scheme was introduced using homomorphic verifiable response method and hash index hierarchy for providing integrity of data. In [11], a novel technique namely, decentralized Cloud Information Accountability was introduced to keep hold of users data in cloud. In [9], allocation scheme was designed keeping in mind the number of clients assigned to the server. In [12], a definition of fairness in congested situation was presented by the author, assuming that multiple resource types are allocated simultaneously to each service request. Next, this work identifies a measure for evaluating fair resource allocation. To make the most efficient use of the resources, [17].The author in [8] presents a method to allocate resources for real-time tasks using the "Infrastructure as a Service "model offered by cloud computing. In [14], decentralized self-adaptation mechanism was designed in order to select among the available resources due to the introduction of Agile Service Networks (ASN).The work in [1] define an architectural framework and principles for energyefficient Cloud computing. The methodology provided in [4] discussed the opportunities and challenges for efficient parallel data processing in clouds and present our research project Nephele. In [16], a new framework that provides efficient green enhancements within a scalable Cloud computing architecture was presented. In [18], Hierarchical Attribute-based solution was designed to provide flexibility and scalability in cloud using cipher-text policy attributed-based encryption scheme for outsourced data in cloud computing environment. In view of the load balancing problem in VM resources scheduling, [6] present a scheduling strategy on load balancing of VM resources based on genetic algorithm. In order to improve resource utilization of large Data Centers while delivering services with higher QoS to Cloud Clients, an automatic resource allocation strategy based on market Mechanism (ARAS-M) is proposed in [13]. A Genetic Algorithm (GA)-based automatic price adjusting algorithm is introduced to deal with the problem of achieving the equilibrium state of ARAS-M is proposed in [13].The work presented in [9] aims to design such a strategy for energy-efficient cloud data centers.

\section{METHODOLOGY}

The survey of research in energy efficient computing is conducted for energy efficient management of clouds, energy efficient resource allocation policies and scheduling algorithms considering QoS expectations and power usage individuality of the devices. A number of open researches confront addressing which bring considerable benefits to both resource provider and customers. The different work involved in "Performance analysis of various architectural approaches in cloud computing environment for energy and bandwidth minimization" is:

\subsection{Energy Conservation in Cloud Infrastructures}

The work in [8] proposed system model that includes two different mechanisms comprising of service request prediction and VM migration. The virtual machine migration worked on the principle that aims to see to that the violation rate related to SLA is minimized. To achieve the objective, slight modifications were required in the existing cloud infrastructure. The cloud infrastructure comprised of a Cloud Controller, Cluster Controller, Storage Controller, and hundreds or thousands of Node Controllers/Servers which are capable of running virtual machines (VMs).The Cloud Controller is an administrative tool for private cloud deployments. The cloud controller in turn manages cloud applications on the basis of the thorough life cycles, comprising of monitoring, metering, and billing. The job of Cluster Controller was to fix the control of VMs running on the nodes and provisioning towards the virtual networking between VMs, and between VMs and external users. The work of the Storage Controller is to provide central storage in a cluster that can be dynamically attached to the VMs. The objective of Node Controller is to make full control towards VM activities that includes execution, inspection and termination of VM instances. The basic assumption of the model was that the cluster controller, cloud controller, storage controller and finally the node controller are placed at similar geographic location.

This work proposes an approach towards achieving energy conservation in existing cloud infrastructures by making use of a service request prediction model. Our model makes use of historical service request data and predicts future request patterns.. The users present in the cloud infrastructure are designed keeping in mind to meet peak load conditions, with provisioning for peak load solved in a rare manner. In such cases most of the period where the clusters in the server remains underutilized. The consumption of energy cloud environment are thus minimized by making use of prediction model using service request and running virtual machines (VMs) across different level of servers. At the prime level, in [8], results shows that if different VMs are executing on the basis of under-utilized servers, a solution for it is to bring several executing VMs altogether to some minimum level of servers results in higher utilization rate by way of sending the other unutilized servers to hibernation, is the way in which the conservation of energy can be achieved. In this work, a modified system model is proposed in which the future service requests from are predicated historical data and identify the number of required active servers at the present time. Any servers remaining are further sent into hibernation point of centrality to measure the hubness that reduces the consumption of energy level present in the cloud infrastructure.

\subsection{Dynamic Resource Allocation for Efficient Parallel Data Processing in the Cloud}

Based on the issues and opportunities presented in the previous section, a novel kind of data processing framework was designed specifically for the cloud environment called as Nephele..Nephele's architecture is a classic master-worker pattern as illustrated in Energy Conservation in Cloud Infrastructures. The objective of the Job Manager(JM) as in [2] is to receive the job related to the client, whose core responsibility is to further schedule them and provide a way towards better 
coordination. The job manager is capable of communicating through the interface where the sole job of the cloud operator is to provide control for instantiation of VMs.The term instances are used in order to further differentiate between VMs with certain hardware characteristics kept in mind. The job of the task manager(TM) as in [2] is to receive with one or more tasks allocated from the job manager at specific instance of time, compiles and executes them, and later on is provided with the completion of task or possibility of errors to the Job Manager. The updated version of instances allocated starts up with a previously compiled VM image. The image is configured to automatically start a Task Manager and register it with the Job Manager. Once all the necessary Task Managers have successfully contacted the Job Manager.

\subsection{Resource Scheduling Strategy Based on Genetic Algorithm in Cloud Computing Environment}

The objective of this work is to find the solution of mapping to the greatest extent according to meet the system load balance or to make the lowest cost of load balancing. Here the best scheduling solution for the current scheduling through is obtained genetic algorithm. The genetic algorithm cost is evaluated through the ratio of present scheduling solution to the optimal best scheduling solution obtained, and finally the scheduling solution is arrived according to the cost gene factor. The solution towards scheduling process is arrived in [6] with the barest level of cost selected as the final solution for scheduling.. In this way, the best strategy is arrived.

\section{Global Scheduling Algorithm}

Considering the Virtual Machine scheduling for resources as discussed in 3.1 in the cloud computing environment and with the advantage of genetic algorithm as illustrated in 3.2, a balanced scheduling strategy of VM resources based on genetic algorithm [6] is presented.. The pseudo code generated is illustrated as below:

\section{Initialization}

Let VM denote virtual machine

Let $\mathrm{S}$ denote scheduling solution set

\section{Repeat}

For each client do

Randomly choose free machine and start scheduling

With (increase in VM) $\boldsymbol{\&} \boldsymbol{\&}$ (increase in time)

For each machine do

Compute load \& variance from $\mathbf{S}$ Endfor

For each solution in S do

Compute best mapping solution // Apply GA

Compute cost divisor for S Endfor

\section{For each $\mathbf{S}$ do}

Select lowest cost

Perform scheduling Endfor

Until (VM need further scheduling)

In every scheduling, the genetic algorithm is used to find the best scheduling solution; and in the next scheduling, because of the accumulation of the best solutions by the original scheduling solutions, the best scheduling solution can always be found to achieve load balancing.

\subsection{Energy-aware allocation of data center resources}

Recent developments related to research in virtualization have resulted in its proliferation across data centers. In the current scenario, the allocation of resources in Cloud environment, data center aims to provide high performance while meeting SLAs, without the main focus towards allocating VMs to minimize energy consumption as presented in [1]. To explore both performance and energy efficiency, three crucial issues were addressed in [1]. To start with, extreme cycling of server in cloud environment results in the less provisioning of reliability. Second, turning resources while not necessary to provide solution towards dynamic environment is highly danger from the point of view of quality of service. Due to the variance related to the workload and consolidation in aggressive nature results in not assignment of resources to certain virtual machines during the peak slot and thereby results in the provisioning of failure towards quality of service. Finally, the third issue discussed in [1] was the big challenges related to SLAs to provide with certain level of accurate application performance management in virtualized environments. All the three issues discussed above require proper and effective consolidation means that can results in the consumption of energy in minimized level at the same time without great compromise for the user-specified QoS requirements.

\section{Architectural framework}

The aim of cloud is to provide a designing for next generation data centers by architecting them as networks of virtual services comprising of software, hardware, database, user-interface, application logic so that the users access and provide certain application level from anywhere in the world on the basis of demand without compromising the costs on the basis of their respective QoS requirements.

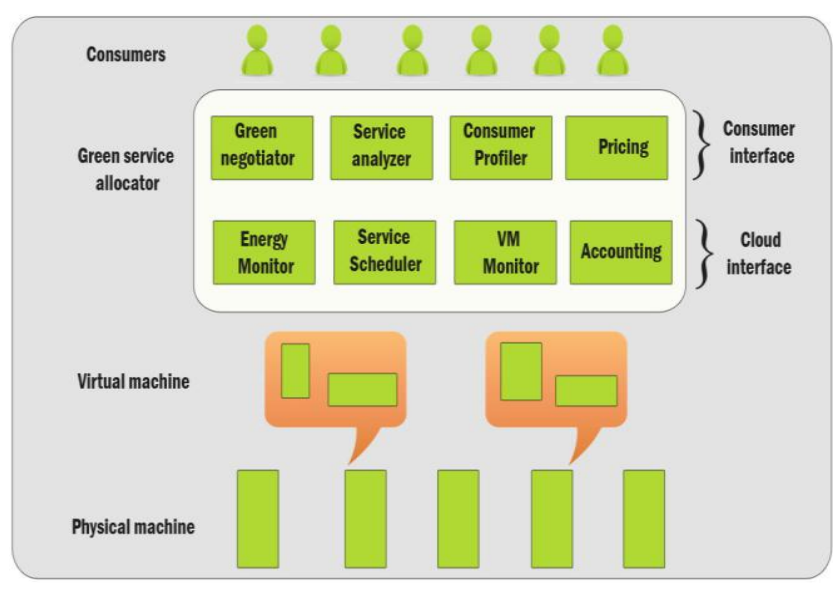

Fig 1: High-level System Architecture

Fig. 1 shows the high-level architecture for supporting energyefficient service allocation in a Green Cloud computing infrastructure. There are basically four main entities involved as discussed in [1]:

1. Consumers/Brokers: The initial task is that the Cloud consumers submit their corresponding service requests from anywhere placed in the world to the Cloud environment.

2. Green Service Allocator: Next, the green service allocator acts as one of the interface between the Cloud infrastructure and cloud consumers. 
(a) Green Negotiator: The green negotiator negotiates with the cloud consumers present in the cloud in order to arrive with the finalization of SLAs with specified level of prices and fixation of penalties

(b) Service Analyzer: The service analyzer work is to interpret and analyze the requirements of services of request submitted before accepting the same for further processing.

(c) Consumer Profiler: The job of consumer profiler as discussed in [1] is to gather specific characteristics of cloud consumers

(d) Pricing: The pricing policy decides a way of how certain level of service requests to be charged in order to manage the supply and demand of resources to be computed in an efficient manner.

(e) Energy Monitor: The job of energy monitor is to monitor the consumption level of energy caused by VMs and the respective physical machines.

(f) Service Scheduler: The service scheduler assigns requests to the corresponding VMs and identifies the resource entitlements for the respective allocated $\mathrm{VMs}$

(g) VMManager: Keeps track of the availability of VMs and their resource usage. It is in charge of provisioning new VMs as well as reallocating VMs across physical machines to adapt the placement.

(h) Accounting: The job of accounting is to monitor the actual usage of resources by VMs and determines for the resource usage costs.

3. VMs: Multiple VMs are started in a dynamic manner and stopped on a single physical machine on the basis of the incoming requests. In [1], multiple VMs concurrently run on the applications on the basis of different operating system environments on a single physical machine. By way of dynamically migrating VMs across physical machines, workloads are stabilized and resources that are not in use is switched to a low-power mode, either switched off or processed in a way so that to operate at low-performance levels resulting in minimization of energy.

4. Physical Machines: The underlying physical computing servers provide the hardware infrastructure for creating virtualized resources to meet service demands.

\section{PERFORMANCE EVALUATION OF VARIOUS RESOURCE ALLOCATION STRATEGIES}

A generic Cloud computing environment, i.e. IaaS, is to evaluate it on a large-scale virtualized data center infrastructure. However, it is extremely difficult to conduct repeatable large-scale experiments on a real infrastructure, which is required to evaluate and compare the proposed resource management algorithms. Therefore, to ensure the repeatability of experiments, simulations have been chosen as a way to evaluate the performance of the proposed heuristics. The CloudSim toolkit has been chosen as a simulation platform as it is a modern simulation framework aimed at Cloud computing environments. In contrast to alternative simulation toolkits (e.g. SimGrid, GangSim), it supports modeling of on-demand virtualizationenabled resource and application management. It has been extended to enable energy-aware simulations as the core framework does not provide this capability. Apart from the energy consumption modeling and accounting, the ability to simulate service applications with workloads variable over time has been incorporated. We have simulated a data center comprising 100 heterogeneous physical nodes. Each node is modeled to have one CPU core with the performance equivalent to 1000,2000 or 3000 MIPS, 8 GB of RAM and 1 TB of storage. Power consumption by the hosts is defined according to the model described in Section 3.2. According to this model, a host consumes from $175 \mathrm{~W}$ with $0 \% \mathrm{CPU}$ utilization, up to 250Wwith $100 \%$ CPU utilization. Each VM requires one CPU core with $250,500,750$ or 1000 MIPS, $128 \mathrm{MB}$ of RAM and 1 GB of storage. The users submit requests for provisioning of 290 heterogeneous VMs that fill the full capacity of the simulated data center. Each VM runs a web-application or any kind of application with variable workload, which is modeled to generate the utilization of CPU according to a uniformly distributed random variable. The application runs for $150,000 \mathrm{MI}$ that is equal to $10 \mathrm{~min}$ of the execution on 250 MIPS CPU with $100 \%$ utilization. Initially, the VMs are allocated according to the requested characteristics assuming 100\% CPU utilization. Each experiment has been run 10 times.

\section{RESULTS AND DISCUSSIONS OF VARIOUS RESOURCE ALLOCATION STRATEGIES}

In order to compare the efficiency of the algorithms we use several metrics to evaluate their performance. The first metric is the total energy consumption by the physical resources of a data center caused by the application workloads. The second performance metric is called the SLA violation percentage, or simply the SLA violations, which is defined as the percentage of SLA violation events relatively to the total number of the processed time frames. An SLA violation is defined as that it occurs when a given VM cannot get the amount of Million Instructions Per Second (MIPS) that are requested. This can happen in cases when VMs sharing the same host require a CPU performance that cannot be provided due to the consolidation. This metric shows the level by which the QoS requirements negotiated between the resource provider and consumers are violated due to the energy-aware resource management. It is assumed that the provider pays a penalty to the client in case of an SLA violation. The third metric is the number of VM migrations initiated by the VM manager during the adaptation of the VM placement. The last performance metric is the average SLA violation, which represents the average CPU performance that has not been allocated to an application when requested, resulting in performance degradation.

Table 5.1. Energy Consumption

\begin{tabular}{|c|c|c|}
\hline \multirow{2}{*}{$\begin{array}{c}\text { Utilization } \\
\text { Threshold } \\
(\%)\end{array}$} & $\begin{array}{c}\text { Energy- } \\
\text { aware } \\
\text { resource } \\
\text { allocation }\end{array}$ & $\begin{array}{c}\text { Energy Optimization } \\
\text { Technique }\end{array}$ \\
\hline 50 & 2.02 & 1.95 \\
\hline 60 & 1.61 & 1.54 \\
\hline 70 & 1.4 & 1.38 \\
\hline 80 & 1.24 & 1.16 \\
\hline 90 & 1.12 & 1.04 \\
\hline 100 & 1.05 & 0.87 \\
\hline
\end{tabular}




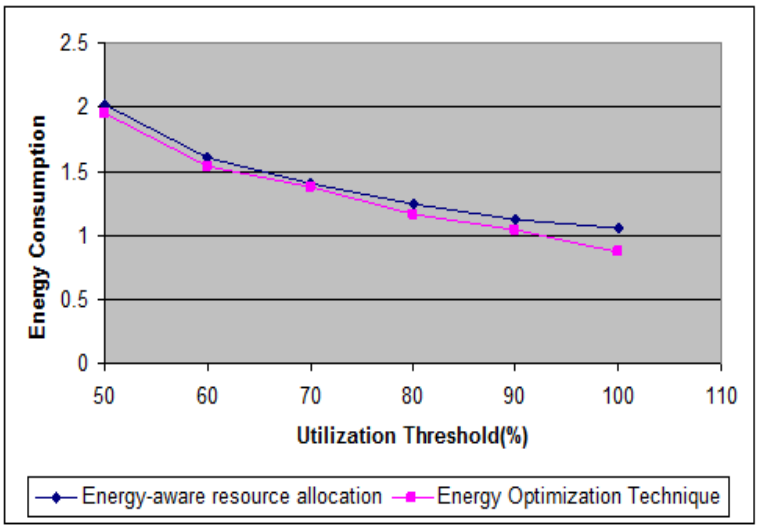

Fig 5.1: Energy Consumption

The results showing the mean energy consumption achieved using Energy-aware resource allocation method and Energy Optimization Technique for different values of the lower utilization threshold and the interval between the thresholds are presented in Fig. 5.1. The graph shows that an increase of the lower utilization threshold leads to decreased energy consumption. However, the low level of energy consumption can be achieved with different intervals between the thresholds. Therefore, to determine the best interval we have to consider another factor, the level of SLA violations. Compared with Energy-aware resource allocation method, Energy Optimization Technique consumes 5 to $9 \%$ less energy.

Table 5.2. Cpu execution time(s)

\begin{tabular}{|c|c|c|c|}
\hline \multirow{2}{*}{$\begin{array}{c}\text { Number } \\
\text { of VMs }\end{array}$} & \begin{tabular}{c}
$|c|$ \\
CPU Execution time(s) \\
\cline { 2 - 4 } \\
Resource \\
Scheduling \\
based on \\
GA
\end{tabular} & $\begin{array}{c}\text { Congestion } \\
\text { Control } \\
\text { method } \\
\text { with fair } \\
\text { Resource } \\
\text { Allocation }\end{array}$ & $\begin{array}{c}\text { Dynamic } \\
\text { resource } \\
\text { allocation } \\
\text { method }\end{array}$ \\
\hline 2 & 0.1 & 0.1 & 0.13 \\
\hline 4 & 0.13 & 0.18 & 0.26 \\
\hline 6 & 0.16 & 0.24 & 0.38 \\
\hline 8 & 0.25 & 0.36 & 0.39 \\
\hline 10 & 0.34 & 0.73 & 0.64 \\
\hline 12 & 0.76 & 0.87 & 0.86 \\
\hline 14 & 1.04 & 1.18 & 1.25 \\
\hline
\end{tabular}

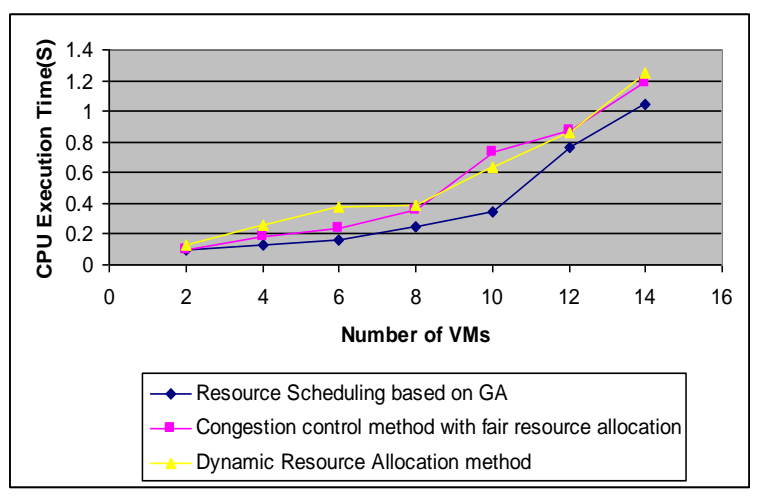

Fig 5.2: CPU Execution time(s)

This work through selecting different number of physical machines and virtual machines and making a larger number of experiments attain CPU execution time of the best solution under different number of VMs. From the Figure 5.2 shown we can see that with the increase of VM number, there is less significant increase of execution time for Resource Scheduling based on GA and it can still keep a good performance, which proves that the efficiency of Resource Scheduling based on GA is 12 to $25 \%$ high.

Table 5.3. VM migrations

\begin{tabular}{|c|c|c|c|}
\hline \multirow{2}{*}{$\begin{array}{c}\text { Number } \\
\text { of VM } \\
\text { started }\end{array}$} & $\begin{array}{c}\text { Energy- } \\
\text { Aware } \\
\text { resource } \\
\text { allocation }\end{array}$ & $\begin{array}{c}\text { Resource } \\
\text { scheduling } \\
\text { based on } \\
\text { GA }\end{array}$ & $\begin{array}{c}\text { Dynamic } \\
\text { resource } \\
\text { allocation } \\
\text { method }\end{array}$ \\
\hline 10 & 2512 & 2847 & 2904 \\
\hline 20 & 3345 & 3976 & 3749 \\
\hline 30 & 3498 & 4026 & 3902 \\
\hline 40 & 3416 & 3992 & 4028 \\
\hline 50 & 3127 & 3690 & 4127 \\
\hline 60 & 3078 & 3414 & 4316 \\
\hline 70 & 3216 & 3857 & 4037 \\
\hline
\end{tabular}

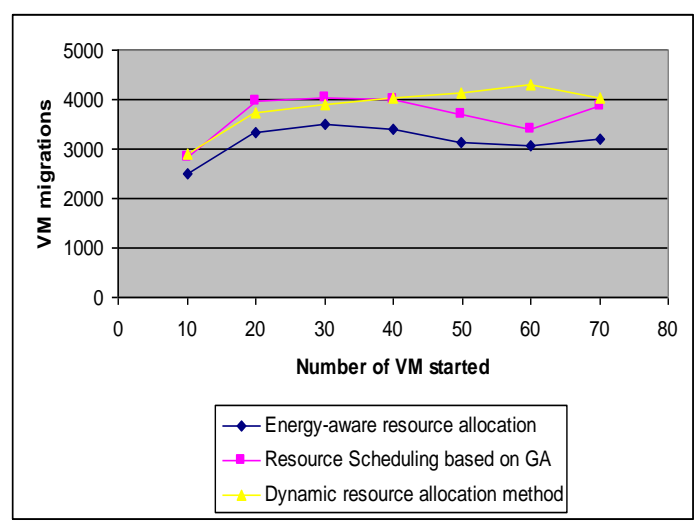

Fig 5.3: VM migrations

On some special occasions, there is a big increase of the load of some nodes in the system due to frequent access thus leads to the load imbalance of the whole system. Under this situation, usually the system cannot realize the system load balancing through only one-time scheduling so it must do it through VM migration. However, the cost of VM migration cannot be neglected. Thus where the VM should be migrated and how to migrate the least number of $\mathrm{VM}$ are also the problems that need consideration during VM scheduling. The algorithm of this work takes historical factors into consideration. It computes the situation of the whole system after scheduling in advance through genetic algorithm and then chooses the scheduling solution with the lowest cost. Figure 5.3 shows the average VM migration ratio while the VM load variation rate $\alpha$ is changing. It can be seen that the method of this work shows conspicuous advantage. The experiment shows that the method of this work can greatly brings down the migration cost.

We investigate the utilization rate of Resource scheduling based on GA, Congestion Control method with fair Resource Allocation, Energy-Aware resource allocation in this work. From the Figure 5.4, we can work out how much resource each model wasted when allocating different number of VMs. And we find that sometimes Energy-Aware resource allocation in this work 
cannot allocate resources for all the VMs even if there are enough resources for the VMs. But Resource scheduling based on GA always gives a good scheduling as long as there are enough resources. On the other hand, we can see that Resource scheduling based on GA in this work saves 13 to $27 \%$ more resources compared with other techniques.

Table 5.4. Utilization rate (\%)

\begin{tabular}{|c|c|c|c|}
\hline \multirow{2}{*}{$\begin{array}{c}\text { Number } \\
\text { of } \\
\begin{array}{c}\text { Started } \\
\text { VM }\end{array}\end{array}$} & $\begin{array}{c}\text { Utilization Rate (\%) } \\
\text { Resource } \\
\text { based on } \\
\text { GA }\end{array}$ & $\begin{array}{c}\text { Congestion Control } \\
\text { method with fair } \\
\text { Resource } \\
\text { Allocation }\end{array}$ & $\begin{array}{c}\text { Energy- } \\
\text { Aware } \\
\text { resource } \\
\text { allocation }\end{array}$ \\
\hline 5 & 69 & 62 & 54 \\
\hline 10 & 72 & 64 & 52 \\
\hline 15 & 64 & 68 & 61 \\
\hline 20 & 67 & 61 & 64 \\
\hline 25 & 69 & 65 & 63 \\
\hline 30 & 73 & 67 & 60 \\
\hline 35 & 75 & 69 & 58 \\
\hline
\end{tabular}

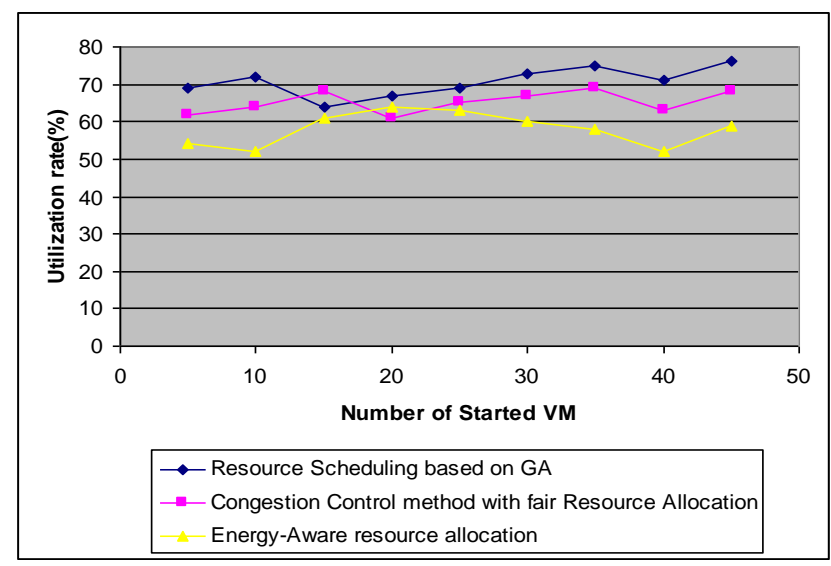

Fig. 5.4. Utilization Rate (\%)

\section{RESEARCH DIRECTIONS}

In all the recent works performed, scheduling and resource allocation is one of the significant aspects to be solved from the user aspect. Parallel processing for analytic tasks is one of the key towards resource allocation but solved at the cost of certain benefits involved and occurrence of overheads. With more complex problems involved, parallelism includes certain level of characterization resulting in constraints. Another interesting problem is to understand the interaction between the scheduler and the capacity controller in green cloud computing. Numerous requests are placed to the cloud at the same time resulting in appropriate allocation of resources to the client whenever there occur an increase in the number of users or total number of data centers. Ensuring the integrity of data in cloud is also a serious concern to be solved. Though the introduction of third party auditor resolves the problem related to integrity towards certain extent, but to be focused when the number of scalability comes into existence. Dynamic allocation of resources directly focuses towards the heterogeneous environment, leaving a greater concern towards the variations in application performance across the resources. The energy conservation in cloud provides with different scheduling system in terms of both power-aware and thermal-aware constraints leading to the maximization of energy savings from the side of physical server. Certain method like genetic algorithm was introduced in order to improve resource utilization to achieve equilibrium. A generic resource manager and plug-in software adaptors can be introduced to permit the interaction with diverse cloud management systems. The model can also extend the machine heterogeneity (i.e.) multiple generation of machines in green cloud computing. It is interesting to expand the development scope from day to multiple years to incorporate the impact of seasonal changes in cloud computing electricity generation and power consumption as well as the predicted changes in various price aspects. Subsequently, one can include the costs of cloud cooling, property purchase and also the cost of renewable energy generation. Finally, it can also take into account more complicated quality-of-service requirements besides limiting the propagation delay in green cloud computing.

From the above listed research directions, the future work may lay foundation to the concentric efforts of providing multitasking based resource scheduler for the cloud computing environment.

\section{CONCLUSION}

Cloud computing technology is gradually more being used in enterprises and business markets. In cloud pattern, an effective resource allocation strategy is required for achieving user fulfillment and also reducing the profit for cloud service providers. This work summarizes the classification of Resource allocation strategies and its impacts in cloud system. Some of the strategies discussed above mainly focus on CPU, memory resources but are lacking in some factors. A review on the various research issues involved in resource allocation strategies in cloud system have been outlined. Research on these issues will lead to promising results, making cloud based applications very popular. Hence this survey work will hopefully motivate future researchers to come up with the minimal energy and bandwidth consumption resource allocation and framework to strengthen the cloud computing paradigm.

\section{REFERENCES}

[1] Anton Beloglazov, Jemal Abawajy, Rajkumar Buyya, "Energy-aware resource allocation heuristics for efficient management of data centers for Cloud computing", Future Generation Computer Systems, pp. 755-768, 2012.

[2] Anusha Reddy, Dr.M.Janga Reddy," Dynamic Resource Allocation and Data Processing Framework for Cloud Architecture" International Journal of Advanced Research in Computer Science and Software Engineering, Volume 2, Issue 10, October 2012

[3] Chandrashekhar S. Pawar and R.B.Wagh," A review of resource allocation policies in cloud computing", Proceedings in World Journal of Science and Technology, 2012

[4] Daniel Warneke and Odej Kao, "Exploiting Dynamic Resource Allocation for Efficient Parallel Data Processing in the Cloud", IEEE Transactions on Parallel and Distributed Systems, January 2011.

[5] Qian Wang, Kui Ren, Wenjing Lou, and Jin Li," Enabling Public Auditability and Data Dynamics for Storage Security in Cloud Computing", IEEE TRANSACTIONS ON PARALLEL AND DISTRIBUTED SYSTEMS, VOL. 22, NO. 5, MAY 2011

[6] Jianhua Gu, Jinhua Hu, Tianhai Zhao, Guofei Sun, “A New Resource Scheduling Strategy Based on Genetic Algorithm 
in Cloud Computing Environment”, Journal Of Computers, vol. 7, no. 1, January 2012.

[7] Jianfeng Yan, Wen-Syan Li,“Calibrating Resource Allocation for Parallel Processing of Analytic Tasks", IEEE International Conference on e-Business Engineering, 2009.

[8] Kumar, K., Jing Feng, Nimmagadda, Y., Yung-Hsiang $\mathrm{Lu}$, "Resource Allocation for Real-Time Tasks Using Cloud Computing", IEEE, Computer Communications and Networks (ICCCN), 2011.

[9] Mehta, A., Menaria, M., Dangi, S., Rao, S., "Energy conservation in cloud infrastructures",IEEE International Conference on Systems Conference (SysCon), 2011.

[10] Olivier Beaumont, Lionel Eyraud-Dubois and Hejer Rejeb,' Heterogeneous Resource Allocation under Degree Constraints", IEEE TRANSACTIONS ON PARALLEL AND DISTRIBUTED SYSTEMS

[11] Smitha Sundareswaran, Anna C. Squicciarini, Member, IEEE, and Dan Lin," Ensuring Distributed Accountability for Data Sharing in the Cloud", IEEE TRANSACTIONS ON DEPENDABLE AND SECURE COMPUTING, VOL. 9, NO. 4, AUGUST 2012

[12] Takuro TOMITA and Shin-ichi KURIBAYASHI, "Congestion control method with fair resource allocation for cloud computing environments",IEEE, 2011.

[13] Xindong You, Jian Wan, Xianghua Xu, Congfeng Jiang, Wei Zhang, Jilin Zhang, "ARAS-M: Automatic Resource Allocation Strategy based on Market Mechanism in Cloud Computing", Journal Of Computers, VOL. 6, NO. 7, JULY 2011.

[14] Vivek Nallur, Rami Bahsoon," A Decentralized SelfAdaptation Mechanism For Service-Based Applications in The Cloud", IEEE TRANSACTIONS ON SOFTWARE ENGINEERING

[15] Yan Zhu, Hongxin Hu, Gail-Joon Ahn, Senior Member, IEEE, Mengyang Yu," Cooperative Provable Data Possession for Integrity Verification in Multi-Cloud
Storage", IEEE TRANSACTIONS ON PARALLEL AND DISTRIBUTED SYSTEMS, Jun 2012

[16] Younge, A.J., von Laszewski, G. ; Lizhe Wang ; LopezAlarcon, S. ; Carithers, W., "Efficient resource management for Cloud computing environments", International Green Computing Conference, 2010.

[17] Zhongni Zheng, Rui Wang, Hai Zhong, Xuejie Zhang, "An approach for cloud resource scheduling based on Parallel Genetic Algorithm", IEEE, 2011.

[18] Zhiguo Wan, Jun'e Liu, and Robert H. Deng," HASBE: A Hierarchical Attribute-Based Solution for Flexible and Scalable Access Control in Cloud Computing", IEEE TRANSACTIONS ON INFORMATION FORENSICS AND SECURITY, VOL. 7, NO. 2, APRIL 2012

[19] V.Vinothina, Dr.R.Sridaran, Dr.PadmavathiGanapathi," A Survey on Resource Allocation Strategies in Cloud Computing", (IJACSA) International Journal of Advanced Computer Science and Applications, Vol. 3, No.6, 2012

\section{AUTHOR'S PROFILE}

N.R. Ram Mohan received the B.E degree from Anna University, Chennai, Tamil Nadu, India, in 2007and M.Tech degree from Manonmaniam Sundaranar University, Trinelveli, Tamil Nadu, India, in 2009. He is currently persuing the Ph.D degree in Computer and Information Technology.Manonmaniam Sundaranar University, Tirunelveli, Tamilnadu, India. His current interest span several areas grid computing, information security and cloud computing environment

E. Baburaj received the M.E. degree in computer science and Engineering from Madurai Kamaraj University, Madurai, Tamil Nadu, India, in 2002 and the Ph.D degree in Computer Science and Engineering from Anna University, Chennai, Tamil Nadu, India, in 2009.

Currently he is a Professor, Dept of Computer Science and Engineering, Sun College of Engineering and Technology, Nagercoil, Tamilnadu, India. His research interest includes Adhoc Networks, Network Security and Cloud Computing. He has published over 20 papers in international journals. 